\title{
Nonalcoholic fatty liver disease and alcohol-related liver disease: From clinical aspects to pathophysiologi- cal insights
}

\author{
Kenichi Ikejima, Kazuyoshi Kon, and Shunhei Yamashina \\ Department of Gastroenterology, Juntendo University Graduate School of Medicine, Tokyo, Japan
}

Two major causes of steatohepatitis are alcohol and metabolic syndrome. Although the underlying causes of alcoholrelated liver disease (ALD) and nonalcoholic fatty liver disease (NAFLD)/nonalcoholic steatohepatitis (NASH) differ, there are certain similarities in terms of the mode of disease progression and underlying pathophysiological mechanisms. Further, excessive alcohol consumption is often seen in patients with metabolic syndrome, and alcoholic hepatitis exacerbation by comorbidity with metabolic syndrome is an emerging clinical problem. There are certain ethnic differences in the development of both NAFLD and ALD. Especially, Asian populations tend to be more susceptible to NAFLD, and genetic polymorphisms in patatin-like phospholipase domain-containing 3 (PNPLA3) play a key role in both NAFLD and ALD. From the viewpoint of pathophysiology, cellular stress responses, including autophagy and endoplasmic reticulum (ER) stress, are involved in the development of cellular injury in steatohepatitis. Further, gutderived bacterial products and innate immune responses in the liver most likely play a profound role in the pathogenesis of both ALD and NASH. Though the recent progress in the treatment of viral hepatitis has reduced the prevalence of viral-related development of hepatocellular carcinoma (HCC), non-viral HCC is increasing. Alcohol and metabolic syndrome synergistically exacerbate progression of steatohepatitis, resulting in carcinogenesis. The gut-liver axis is a potential therapeutic and prophylactic target for steatohepatitis and subsequent carcinogenesis. (Clin Mol Hepatol 2020;26:728-735)

Keywords: Alcohol-related liver disease; Non-alcoholic fatty liver disease; Stress responses; Immunity, Innate; Gut microbiota

\begin{abstract}
Abbreviations:
ADH, alcohol dehydrogenase; ALD, alcohol-related liver disease; ALDH, aldehyde dehydrogenase; APOC3, apolipoprotein $\mathrm{C}$; BiP, binding immunoglobulin protein; CHOP, C/EBP homologous protein; CVD, cardiovascular disease; CYP, cytochrome P450; ER, endoplasmic reticulum; EtOH, ethanol; HCC, hepatocellular carcinoma; IP3R, inositol trisphosphate receptor; LC, liver cirrhosis; LPS, lipopolysaccharide; NAFL, nonalcoholic fatty liver; NAFLD, nonalcoholic fatty liver disease; NASH, nonalcoholic steatohepatitis; NK, natural killer; PAMP, pathogen-associated molecular pattern; PNPLA3, patatin-like phospholipase domain-containing 3; RFX, rifaximin; ROS, reactive oxygen species; SIBO, small intestinal bacterial overgrowth; SNP, single nucleotide polymorphism; SXBP1, spliced forms of X-box-binding protein-1; TM6SF2, transmembrane 6 superfamily member 2; UPR, unfolded protein response; $\mathrm{UXBP1}$, un-spliced of X-box-binding protein-1
\end{abstract}

\section{Corresponding author : Kenichi Ikejima}

Department of Gastroenterology, Juntendo University Graduate School of Medicine, 2-1-1, Hongo, Bunkyo-ku, Tokyo 113-8421, Japan

Tel: +81-3-3813-3111, Fax: +81-3-3813-8862

E-mail: ikejima@juntendo.ac.jp

https://orcid.org/0000-0002-2657-0840 


\section{INTRODUCTION}

Nonalcoholic fatty liver disease (NAFLD) is a clinical entity comprising a wide spectrum of liver diseases from nonalcoholic fatty liver (NAFL) to nonalcoholic steatohepatitis (NASH), a progressive form of chronic liver disease eventually resulting in liver cirrhosis (LC) and hepatocellular carcinoma (HCC)., ${ }^{1,2}$ The term NAFLD is the counterpart of alcohol-related (-associated) liver disease (ALD), which suggests that this clinico-pathological entity is heterogeneous, while excluding liver disease attributable to excessive alcohol consumption and other major etiologies such as viral hepatitis and autoimmunity. Most NAFLD is closely related to metabolic syndrome; therefore, NAFLD is now widely accepted as a liver manifestation of metabolic syndrome. Since liver is one of the key organs for the systemic regulation of metabolism, ${ }^{5}$ pathophysiological changes in the liver not only result in the progression of chronic liver disease, but also influence various systemic conditions, especially the development of atherosclerotic diseases. ${ }^{6}$

The obvious and fundamental difference between NAFLD and ALD lies in the amount of alcohol consumption, and the clinical features of typical ALD are distinct from those of NAFLD, even though these two disease entities share similar pathological findings and pathophysiology. Recently, however, a considerable proportion of patients with ALD have also come to demonstrate comorbid metabolic syndrome as well. ${ }^{7}$ Since the differential diagnosis of NAFLD and ALD is based solely on the level of alcohol intake, the combination of ALD and metabolic syndrome often causes confusion and difficulty in the clinical setting. Further, a portion of the patients likely to be diagnosed with NAFLD/NASH may be occasional drinkers, and/or even former heavy-drinkers. Given the lines of evidence indicating potential pathophysiological similarities between ALD and NAFLD/NASH, great attention should be paid to synergistic worsening of ALD in combination with metabolic syndrome.

\section{NAFLD AND ALD IN ASIA}

Metabolic syndrome is highly prevalent in industrialized Western countries, ${ }^{8-10}$ and is also a growing health problem in Asian countries. ${ }^{11,12}$ Further, the susceptibility to obesity, diabetes, and cardiovascular disease (CVD) differs among various ethnic groups. ${ }^{13-15}$ Thus, increasing attention is being paid to NAFLD/ $\mathrm{NASH}$, not only in the industrialized countries of the West but also in the East, with growing concern even in developing countries. ${ }^{16,17}$
It is widely believed that the Western life-style, especially the Western diet, is one of the leading causes of metabolic syndrome and NAFLD. ${ }^{18}$ In Asian countries, therefore, westernization of the diet and industrialization most likely have led to the increase in the prevalence of NAFLD. ${ }^{17}$ The traditional Japanese diet (Washoku), which favors fish over meat dishes and rich in soybean products, is thought to be healthy and sustainable dietary patterns. ${ }^{19}$ Thus, typical Japanese cuisine is believed to be protective against development of NAFLD.

On the other hand, Asian populations demonstrate higher susceptibility to diabetes, even though obesity levels are lower than in Western people. ${ }^{20,21}$ It has also been reported that the prevalence of non-obese NAFLD is higher in Asian countries. ${ }^{22}$ Thus, it seems likely that the prevalence of NAFLD/NASH will continue to rise if the westernization of life-style progresses further in developing countries in Asia.

In terms of alcohol intake, per capita consumption values are lower in Asian countries compared to developed countries in the West. ${ }^{23}$ This disparity is partly due to ethnic differences in polymorphisms in ethanol (EtOH)-metabolizing enzymes. ${ }^{24}$ In Asian populations, however, heterozygosity for the aldehyde dehydrogenase $(A L D H) 2^{*} 2$ allele, which result in lower ALDH2 activity, is highly prevalent at around $40-50 \% .{ }^{25}$ Such individuals need attention since they might suffer more severe liver injury following heavy drinking.

\section{GENDER DIFFERENCES IN NAFLD AND ALD}

There is overt gender difference in the development of ALD: women are more susceptible to ALD than men. ${ }^{26}$ It is well-known that women develop more severe ALD even after consuming less alcohol over a shorter period. ${ }^{27}$ The female predominance in ALD has also been demonstrated in animal models with equal $\mathrm{EtOH}$ loads on a body weight basis, ${ }^{28,29}$ indicating that the phenomenon is not a simple difference in body mass and/or liver volume as suggested in human studies. ${ }^{30,31}$ In contrast, any gender difference in NAFLD is variable, and uncertain. Though some pioneering studies had suggested female predominance in NAFLD, the following population-based studies, especially based on the third NHANES data, have demonstrated a higher prevalence of NAFLD in male subjects. ${ }^{32}$ In animal models, some reports indicated that male rodents develop more severe steatohepatitis than females. ${ }^{33,34}$

With regard to the underlying mechanism of gender differences 
in ALD and NASH, it has been proposed that estrogen plays a pivotal role, possibly in different ways. It has been demonstrated that ovariectomy prevents liver injury following long-term EtOH administration in rodents, whereas estrogen replacement reverses the phenomenon, indicating that estrogen exacerbates alcoholinduced liver injury. ${ }^{29}$ In contrast, estrogen has been suggested to play a preventive role in non-alcoholic models of steatohepatitis in rodents. ${ }^{33}$ These findings are consistent with the clinical observations that women at young ages develop milder NAFLD than men, while NAFLD in women is more prevalent in those who past menopause. ${ }^{35,36}$

\section{POTENTIAL MECHANISTIC INSIGHTS IN THE PATHOGENESIS OF NAFLD AND ALD}

\section{Relationship between metabolic syndrome and fatty liver/steatohepatitis}

In the relationship between metabolic diseases and NAFLD, causality may not be simply unidirectional. Epidemiological studies have suggested positive correlations between the prevalence of metabolic phenotypes and NAFLD/NASH, leading to the hypothesis that pathophysiological changes in the liver initiate systemic alterations in metabolism, thereby exaggerating atherosclerotic diseases. It is also conceivable that systemic micro-inflammation triggers insulin resistance, vascular damage, and NAFLD/NASH, simultaneously. To some extent, a fatty liver per se may be benign or even protective against systemic metabolic disorders. Fat accumulation in the liver is a physiological response to dietary overload, preventing over-flux of excess nutrients into the systemic circulation. Thus, it can be argued that fat accumulation in the liver, which is induced by hyperinsulinemia, would serve as a buffer to protect from, or delay, the onset of overt diabetes. The capacity of fat storage in the liver, therefore, may be critical for the progression of systemic metabolic disorders. Moreover, emerging lines of evidence suggest a possible sub-classification of NAFLD based on genetic variants, which would predict an atherosclerotic phenotype distinct from progressive liver disease. In summary, there is a close relationship between metabolic diseases and NAFLD/NASH, while the pathophysiological mechanisms remain to be explored in greater detail.

\section{Genetic background in NAFLD and ALD}

Since NAFLD is a heterogeneous amalgamation of fatty liver diseases, genetic predisposing factors seem to be diverse as is true for other metabolic syndrome phenotypes such as diabetes and dyslipidemia. The heritability of NAFLD has been estimated to be around $39 \%$ in a familial aggregation study, ${ }^{37}$ though the influence of environmental factors cannot be entirely excluded.

A genome-wide association study revealed that a single nucleotide polymorphism (SNP) of the patatin-like phospholipase domain-containing 3 (PNPLA3) gene is associated with susceptibility to NAFLD. ${ }^{38}$ The $1148 \mathrm{M}$ variant of the PNPLA3 gene is associated with the development of fatty liver in both alcoholic and nonalcoholic individuals. Although it is likely that the genetic variation of PNPLA3 is not associated with other manifestations of metabolic syndrome, there is a certain link between PNPLA3 gene polymorphisms and ethnicity. ${ }^{38,39}$ Association of PNPLA3 variants with NAFLD has also been shown in Asian populations in China, ${ }_{1}^{40} \mathrm{Ja}$ pan, ${ }^{41}$ and Korea. ${ }^{42,43}$ Aside from PNPLA3, variants in other genes have been identified as genetic risk factors for NAFLD/NASH, including transmembrane 6 superfamily member 2 (TM6SF2) and apolipoprotein C (APOC3), etc. ${ }^{44}$

Notably, PNPLA3 variants are also a significant genetic risk for ALD. ${ }^{45}$ Though a variety of EtOH-metabolizing enzymes show gene polymorphisms, which are closely related to addictive phenotypes and the ability take in EtOH, PNPLA3 gene polymorphism has been shown to be the most critical risk for progressive organ injury.

\section{Lipid dynamics, EtOH metabolism and formation of a fatty liver}

In the process of fatty liver development, regardless of metabolic background or alcohol intake, impaired balance among uptake, synthesis, catabolism, and production/release results in fat accumulation in hepatocytes. A variety of regulatory molecules contribute to lipid dynamics in the liver (Fig. 1). Ethanol is mainly metabolized in hepatocytes through two major enzymes, alcohol dehydrogenase (ADH) and cytochrome P450 (CYP) 2E1. In addition to redox shift by $\mathrm{EtOH}$ metabolism, reactive oxygen species (ROS) generated by CYP2E1, as well as from damaged mitochondria, is involved in oxidative cellular injury. ${ }^{46}$ Redox shift by increasing the NADH/NAD ratio is known to suppress gluconeogenesis. Inhibition of $\beta$-oxidation is also an important step in ethanol-induced lipid accumulation in hepatocytes (Fig. 2). 


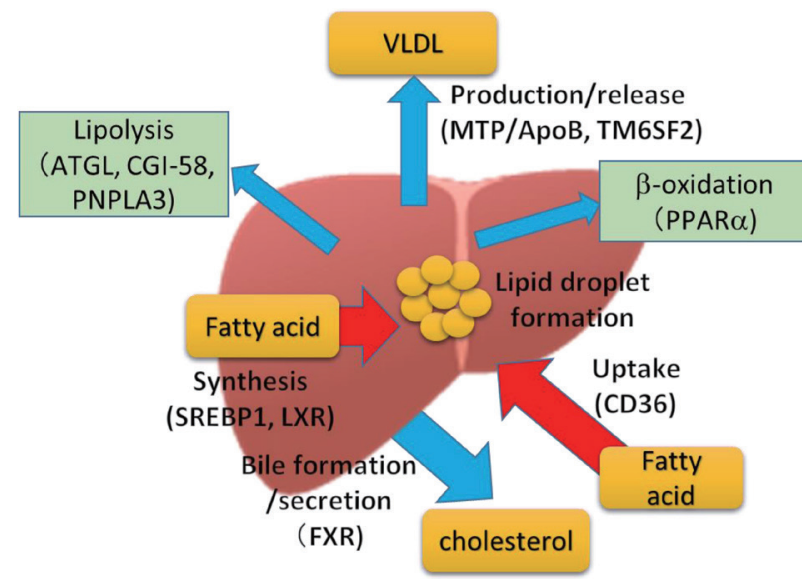

Figure 1. Impaired balance among uptake, synthesis, catabolism, and production/release results in fat accumulation in hepatocytes. VLDL, very low density lipoprotein; ATGL, adipose triglyceride lipase; CGI-58, comparative gene identification 58; PNPLA3, patatin-like phospholipase domain-containing 3; MTP, microsomal triglyceride transfer protein; ApoB, apolipoprotein B; TM6SF2, transmembrane 6 superfamily member 2; PPARa, peroxisome proliferator-activated receptor a; SREBP1, sterol regulatory element binding transcription factor $1 ; L X R$, liver $X$ receptor; CD36, cluster of differentiation 36; FXR, farnesoid $X$ receptor.

\section{Cellular stress responses in NAFLD and ALD}

Cellular stress responses protect the cell, the organ and/or organism from toxic stimuli; however, inappropriate and/or overzealous responses contribute to disease pathogenesis. Indeed, there is significant crosstalk between stress responses and the innate immune response that may contribute to the initiation and progression of disease pathogenesis. The biochemical impact of steatohepatitis drives alterations in substrate supply and metabolism in the liver. For instance, lysosomal-degradation of unnecessary or dysfunctional cellular components is key not only for maintaining cellular energy levels during starvation, but also for an adequate response of this organ to stressors. ALD/NASH both impair autophagy, ${ }^{47}$ which is implicated in the pathogenesis of these diseases.

The endoplasmic reticulum (ER) is a multifunctional organelle required for the regulation of calcium homeostasis, lipid metabolism, and protein synthesis. A number of cellular stress conditions lead to the accumulation of unfolded or misfolded proteins in the ER and disruption of ER homeostasis, which can trigger ER stress. ER stress activates the unfolded protein response (UPR). The UPR pathway includes induction of several molecular chaperones that restore cellular homeostasis by promoting the folding or degradation of unfolded proteins; however, if ER stress is prolonged or too

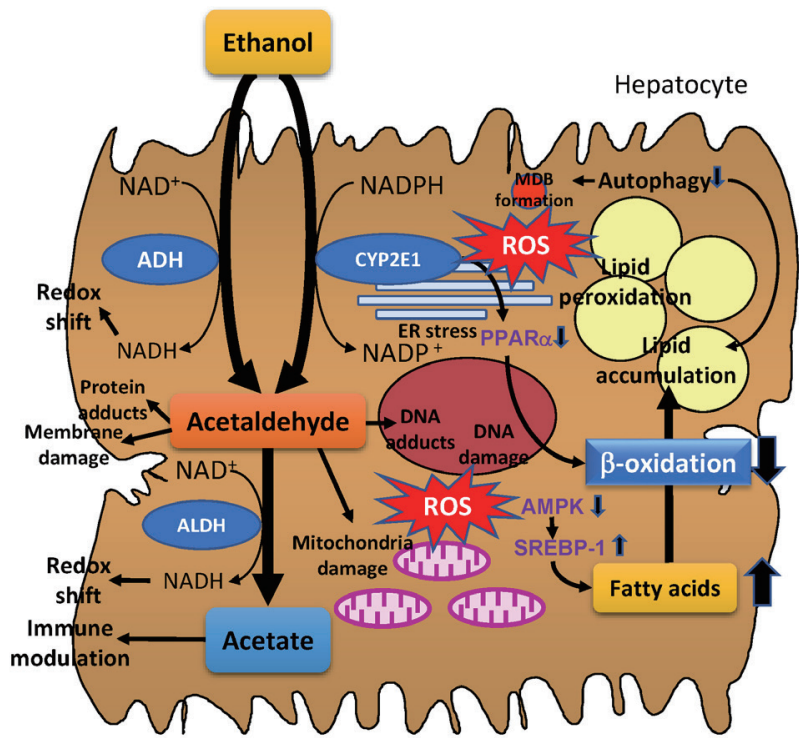

Figure 2. Ethanol metabolism and lipid accumulation in hepatocytes. $\mathrm{NAD}^{+}$, nicotinamide adenine dinucleotide ${ }^{+}$; NADPH, nicotinamide-adenine dinucleotide phosphate; $\mathrm{MDB}$, mallory denk body; $\mathrm{ADH}$, alcohol dehydrogenase; CYP2E1, cytochrome P450 2E1; ROS, reactive oxygen species; ER, endoplasmic reticulum; $A L D H$, aldehyde dehydrogenase; AMPK, AMP-activated protein kinase; SREBP-1, sterol regulatory element binding transcription factor-1.

severe, the signaling switches from pro-survival to pro-death, leading to ER stress-induced apoptosis. Several studies have shown that ER stress contributes to the development of ALD. ${ }^{48}$

To investigate the comorbidity of ALD and metabolic syndrome, we have recently applied the mouse model of chronic-binge EtOH liver injury (NIAAA model) to obese KK-Ay mice. ${ }^{49}$ Chronic-plusbinge $\mathrm{EtOH}$ intake induced massive hepatic steatosis along with hepatocyte apoptosis and inflammation, and increased ER stress markers including binding immunoglobulin protein (BiP), unspliced and spliced forms of X-box-binding protein-1 (UXBP1 and sXBP1, respectively), inositol trisphosphate receptor (IP3R), and C/EBP homologous protein (CHOP), and also enhanced the oxidative stress markers heme oxygenase- 1 and 4-hydroxynonenal. Administration of the chemical chaperone 4-phenylbutyric acid during chronic EtOH exposure ameliorated steatohepatitis after chronic-binge EtOH, and completely inhibited both ER and oxidative stress markers. These findings indicated that binge EtOH intake after chronic consumption induces massive ER stress-related oxidative stress followed by liver injury, and inhibition of ER stress using a chemical chaperone is a potential preventive therapy for alcoholic liver injury especially in obese subjects. 


\section{CLINICAL and MOLECULAR}

\section{HEPATOLOGY}

Volume_26 Number_4 October 2020

\section{Gut microbiota in steatohepatitis due to metabolic syndrome and alcohol}

Increasing attention has been paid to the gut microbiota in human health and disease. ${ }^{50}$ Indeed, the gut microbiota is dynamically altered by dietary factors, lifestyle, and alcohol intake. Gut microbiota-dependent activation of hepatic innate immunity is important in the pathogenesis of steatohepatitis caused by both alcohol and metabolic syndrome (Fig. 3). Chronic alcohol exposure, as well as dietary overload, compromises gut barrier function causing increases in intestinal permeability, thereby aggravating translocation of bacterial products into the portal blood. ${ }^{51}$ Pathogen-associated molecular patterns (PAMPs) derived from gut microbiota elicit production and release of inflammatory cytokines through multiple innate immune-signaling pathways, resulting in the exacerbation of steatohepatitis. ${ }^{52}$

We investigated the alteration in the small intestinal microbiota profile following chronic EtOH feeding in $\mathrm{KK}-\mathrm{A}^{\mathrm{y}}$ mice, and the effect of rifaximin (RFX) on liver injury following chronic/binge administration of EtOH. ${ }^{53}$ Treatment with RFX significantly suppressed hepatic steatosis, as well as the increase in oxidative stress and inflammatory cytokines, in $\mathrm{KK}-\mathrm{A}^{\mathrm{y}}$ mice given $\mathrm{EtOH}$ feeding/binge. Chronic EtOH feeding increased the net amount of small intestinal bacteria, but RFX did not prevent this increase. At the bacterial order level, however, EtOH dramatically increased the relative abundance of the Erysipelotrichales, whose increase in RFX-treated mice was drastically substituted for by the Bacteroidales. Small intestinal bacterial overgrowth (SIBO) and leaky gut

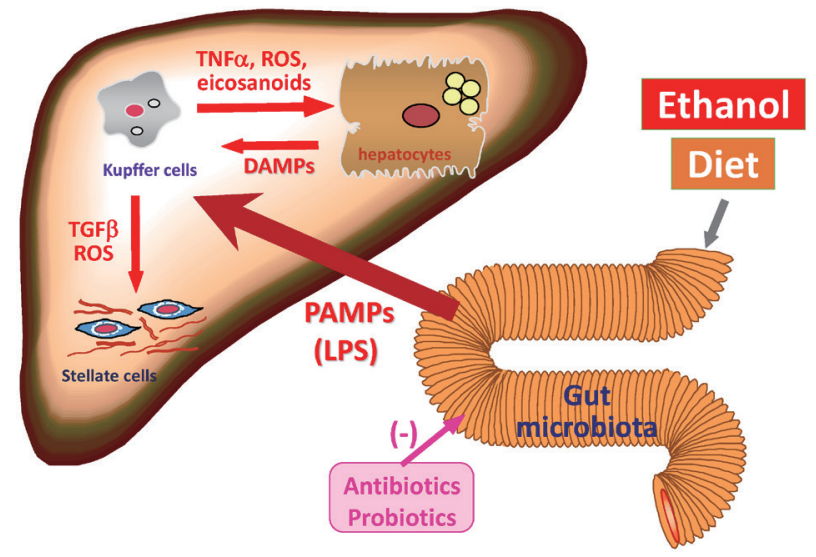

Figure 3. Gut microbiota-dependent activation of hepatic innate immunity caused by both alcohol and metabolic syndrome in the pathogenesis of steatohepatitis. TNF, tumor necrosis factor; ROS, reactive oxygen species; DAMP, damage- associated molecular pattern; PAMP, pathogen-associated molecular pattern; LPS, lipopolysaccharide. result in the increase in bacteria-derived substances such as endotoxin (lipopolysaccharide [LPS]) in the portal blood, which elicit innate immune responses in the liver. Thus, it has been postulated that the modulation of small intestinal microbiota is critical for the prevention of alcoholic liver injury in the context of comorbid metabolic syndrome.

More recently, it has been demonstrated that Candidalysin, a fungal exotoxin, also contributes to the exacerbation of alcoholic hepatitis. ${ }^{54}$ It has been reported that fecal levels of Candida albicans and endothelin-converting enzyme 1 are increased in patients with alcoholic hepatitis. ${ }^{55}$ Candidalysin enhances ethanolinduced liver disease and is associated with higher mortality in mice. Indeed, Candidalysin damages hepatocytes in a dose-dependent manner. Moreover, Candidalysin is also associated with mortality and the severity of liver disease in patients with alcoholic hepatitis. The variety of bacterial and fungal components and products closely associated with the pathophysiology of steatohepatitis merit deep interest.

\section{Innate immunity and ALD/NAFLD}

It has been shown that metabolic reprogramming of hepatic cells during steatohepatitis is not relegated solely to hepatocytes, but hepatic macrophages also undergo metabolic reprogramming during ALD/NAFLD, which may contribute to polarizing the phenotype of the macrophages to a proinflammatory state. Importantly, PAMPs released from the GI tract activate the innate immune response in liver (Fig. 3). Indeed, innate immune responses appear to be primed for stimulus by PAMPs during ALD/NAFLD. Liver also contains other types of innate immune cells, including natural killer (NK) and NKT cells. ${ }^{56}$ Both EtOH and diet alter the expression patterns of hepatic NK and NKT cells, which dysregulates not only the Th1/Th2 balance in hepatic microenvironment, but also immune surveillance against carcinogenesis. ${ }^{57}$ Therefore, maintaining intestinal barrier integrity and the balance of gut microbiota appears to be key in preventing ALD/NAFLD.

\section{ALD/NAFLD AND HCC}

Epidemiological surveys have revealed that the proportion of HCC based on non-viral chronic liver diseases is gradually increasing in Japan, although the majority of HCC develops in patients with chronic viral hepatitis $C$ and $B .{ }^{58}$ However the contribution of ALD is far from negligible, accounting for almost half of non-viral 
HCC. Since metabolic syndrome-related NASH appears to be increasing, NASH can be predicted to take the place of viral hepatitis as the major cause of HCC even in Asian countries including Japan in the foreseeable future.

Nowadays, we sometimes encounter patients with NASH bearing large HCC tumors, due to the lack of proper screening strategies ${ }^{59}$ In screening fatty liver individuals for HCC, one of the critical disadvantages is the poor diagnostic value of ultrasound examination. Magnetic resonance imaging is a preferable technique for detection of tumorous lesions in fatty liver; however, it is less cost-effective and not suitable for mass-screening.

From the viewpoint of pathophysiology, the mechanisms underlying hepatic carcinogenesis in steatohepatitis most likely involve metabolic abnormalities including altered sensitivity to insulin, excessive cellular stress/damage responses, and impaired immune surveillance. Further, emerging roles for bacterial metabolites including short-chain fatty acids and secondary bile acids in metabolic modulation and carcinogenesis have been proposed, ${ }^{60,61}$ suggesting potential prophylactic/therapeutic targets.

\section{EXTRAHEPATIC CARCINOGENESIS IN ALD AND NAFLD/NASH}

Besides HCC, both alcohol and metabolic syndrome have also been demonstrated increase the risk of cancer development in extrahepatic organs. The affected organs, however, show certain differences and similarities between ALD and metabolic syndrome-based NAFLD. ALD patients, especially those drinking beverages of higher alcohol content, often develop esophageal cancer, as well as head and neck tumors. ${ }^{62,63}$ In contrast, esophageal cancer is less frequent in NAFLD individuals, while increases in waist circumference is associated with increased risk of Barrett cancer. ${ }^{64}$ On the other hand, both alcohol and diabetes are overt risk factors for pancreatic cancer. ${ }^{65}$ Similarly, both higher alcohol intake and obesity/NAFLD have been reported as risk factors for cancers of the colon and breast. ${ }^{66,67}$ It is therefore important to pay attention to systemic screening for cancer in patients of both ALD and NAFLD/NASH.

\section{CONCLUSIONS}

NAFLD is now the most prevalent chronic liver disease, especially in developed countries worldwide. NASH, a progressive form of NAFLD, also gives rise to HCC in its advanced stages. It is of note that alcohol intake, even in moderate levels, synergistically worsens metabolic steatohepatitis. Since ALD and NASH share a common pathophysiological basis involving the gut-liver axis, alterations in the gut microbiota (dysbiosis) and subsequent changes in a variety of microbiota-derived components/metabolites most likely contribute to the synergistic actions of alcohol and metabolic factors in the progression of steatohepatitis and carcinogenesis.

\section{Acknowledgements}

The authors thank Dr. Robert F. Whittier (Project Professor, Division of Medical Education, Juntendo University School of Medicine) for English proofreading and critical advice on the manuscript.

\section{Conflicts of Interest}

The authors have no conflicts to disclose.

\section{REFERENCES}

1. Angulo P. Nonalcoholic fatty liver disease. N Engl J Med 2002;346: 1221-1231.

2. Torres DM, Harrison SA. Diagnosis and therapy of nonalcoholic steatohepatitis. Gastroenterology 2008;134:1682-1698.

3. European Association for the Study of the Liver. EASL clinical practice guidelines: management of alcohol-related liver disease. J Hepatol 2018;69:154-181.

4. Crabb DW, Im GY, Szabo G, Mellinger JL, Lucey MR. Diagnosis and treatment of alcohol-associated liver diseases: 2019 practice guidance from the American Association for the Study of Liver Diseases. Hepatology 2020;71:306-333.

5. Eckel RH, Grundy SM, Zimmet PZ. The metabolic syndrome. Lancet 2005;365:1415-1428.

6. Byrne CD, Targher G. NAFLD: a multisystem disease. J Hepatol 2015;62:S47-S64.

7. Chiang DJ, McCullough AJ. The impact of obesity and metabolic syndrome on alcoholic liver disease. Clin Liver Dis 2014;18:157-163.

8. Cameron AJ, Shaw JE, Zimmet PZ. The metabolic syndrome: prevalence in worldwide populations. Endocrinol Metab Clin North Am 2004;33:351-375.

9. Ford ES, Li C, Zhao G. Prevalence and correlates of metabolic syndrome based on a harmonious definition among adults in the US. J Diabetes 2010;2:180-193.

10. Aguilar M, Bhuket T, Torres S, Liu B, Wong RJ. Prevalence of the metabolic syndrome in the United States, 2003-2012. JAMA 
2015;313:1973-1974.

11. Gu D, Reynolds K, Wu X, Chen J, Duan X, Reynolds RF, et al. Prevalence of the metabolic syndrome and overweight among adults in China. Lancet 2005;365:1398-1405.

12. Pan WH, Yeh WT, Weng LC. Epidemiology of metabolic syndrome in Asia. Asia Pac J Clin Nutr 2008;17 Suppl 1:37-42.

13. Lee CM, Huxley RR, Woodward M, Zimmet P, Shaw J, Cho NH, et al. Comparisons of metabolic syndrome definitions in four populations of the Asia-Pacific region. Metab Syndr Relat Disord 2008;6:37-46.

14. Gurka MJ, Lilly CL, Oliver MN, DeBoer MD. An examination of sex and racial/ethnic differences in the metabolic syndrome among adults: a confirmatory factor analysis and a resulting continuous severity score. Metabolism 2014;63:218-225.

15. Falkner B, Cossrow ND. Prevalence of metabolic syndrome and obesity-associated hypertension in the racial ethnic minorities of the United States. Curr Hypertens Rep 2014;16:449.

16. Farrell GC. Non-alcoholic steatohepatitis: what is it, and why is it important in the Asia-Pacific region? J Gastroenterol Hepatol 2003;18:124-138.

17. Ching-Yeung Yu B, Kwok D, Wong VW. Magnitude of nonalcoholic fatty liver disease: Eastern perspective. J Clin Exp Hepatol 2019;9:491-496.

18. Romero-Gómez M, Zelber-Sagi S, Trenell M. Treatment of NAFLD with diet, physical activity and exercise. J Hepatol 2017;67:829-846.

19. Gabriel AS, Ninomiya K, Uneyama $H$. The role of the Japanese traditional diet in healthy and sustainable dietary patterns around the world. Nutrients 2018;10:173.

20. Kodama K, Tojjar D, Yamada S, Toda K, Patel CJ, Butte AJ. Ethnic differences in the relationship between insulin sensitivity and insulin response: a systematic review and meta-analysis. Diabetes Care 2013;36:1789-1796.

21. Rhee EJ. Diabetes in Asians. Endocrinol Metab (Seoul) 2015;30:263269.

22. Liu CJ. Prevalence and risk factors for non-alcoholic fatty liver disease in Asian people who are not obese. J Gastroenterol Hepatol 2012;27:1555-1560.

23. Liangpunsakul $S$, Haber $P$, McCaughan GW. Alcoholic liver disease in Asia, Europe, and North America. Gastroenterology 2016;150:17861797.

24. Wall TL, Luczak SE, Hiller-Sturmhöfel S. Biology, genetics, and environment: underlying factors influencing alcohol metabolism. Alcohol Res 2016;38:59-68.

25. Eng MY, Luczak SE, Wall TL. ALDH2, ADH1B, and ADH1C genotypes in Asians: a literature review. Alcohol Res Health 2007;30:22-27.

26. O'Shea RS, Dasarathy S, McCullough AJ; Practice Guideline Committee of the American Association for the Study of Liver Diseases; Practice Parameters Committee of the American College of Gastroenterology. Alcoholic liver disease. Hepatology 2010;51:307-328.
27. Becker U, Deis A, Sørensen TI, Grønbaek M, Borch-Johnsen K, Müller $C F$, et al. Prediction of risk of liver disease by alcohol intake, sex, and age: a prospective population study. Hepatology 1996;23:10251029.

28. Kono H, Wheeler MD, Rusyn I, Lin M, Seabra V, Rivera CA, et al. Gender differences in early alcohol-induced liver injury: role of CD14, NF-kappaB, and TNF-alpha. Am J Physiol Gastrointest Liver Physiol 2000;278:G652-G661.

29. Yin M, Ikejima K, Wheeler MD, Bradford BU, Seabra V, Forman DT, et al. Estrogen is involved in early alcohol-induced liver injury in a rat enteral feeding model. Hepatology 2000;31:117-123.

30. Kwo PY, Ramchandani VA, O'Connor S, Amann D, Carr LG, Sandrasegaran K, et al. Gender differences in alcohol metabolism: relationship to liver volume and effect of adjusting for body mass. Gastroenterology 1998;115:1552-1557.

31. Dettling A, Fischer F, Bohler S, Ulrichs F, Skopp G, Graw M, et al. Ethanol elimination rates in men and women in consideration of the calculated liver weight. Alcohol 2007;41:415-420.

32. Mishra A, Younossi ZM. Epidemiology and natural history of nonalcoholic fatty liver disease. J Clin Exp Hepatol 2012;2:135-144.

33. Matsushita N, Hassanein MT, Martinez-Clemente M, Lazaro R, French SW, Xie W, et al. Gender difference in NASH susceptibility: roles of hepatocyte Ikk $\beta$ and Sult1e1. PLoS One 2017;12:e0181052.

34. Marin V, Rosso N, Dal Ben M, Raseni A, Boschelle M, Degrassi C, et al. An animal model for the juvenile non-alcoholic fatty liver disease and non-alcoholic steatohepatitis. PLoS One 2016;11:e0158817.

35. Lonardo A, Nascimbeni F, Ballestri S, Fairweather D, Win S, Than TA, et al. Sex differences in nonalcoholic fatty liver disease: state of the art and identification of research gaps. Hepatology 2019;70:14571469.

36. Chen KL, Madak-Erdogan Z. Estrogens and female liver health. Steroids 2018;133:38-43.

37. Schwimmer JB, Celedon MA, Lavine JE, Salem R, Campbell N, Schork NJ, et al. Heritability of nonalcoholic fatty liver disease. Gastroenterology 2009;136:1585-1592.

38. Romeo S, Kozlitina J, Xing C, Pertsemlidis A, Cox D, Pennacchio LA, et al. Genetic variation in PNPLA3 confers susceptibility to nonalcoholic fatty liver disease. Nat Genet 2008;40:1461-1465.

39. Chen LZ, Xin YN, Geng N, Jiang M, Zhang DD, Xuan SY. PNPLA3 I148M variant in nonalcoholic fatty liver disease: demographic and ethnic characteristics and the role of the variant in nonalcoholic fatty liver fibrosis. World J Gastroenterol 2015;21:794-802.

40. Li X, Zhao Q, Wu K, Fan D. I148M variant of PNPLA3 confer increased risk for nonalcoholic fatty liver disease not only in European population, but also in Chinese population. Hepatology 2011;54:2275.

41. Kawaguchi T, Sumida Y, Umemura A, Matsuo K, Takahashi M, Takamura $T$, et al. Genetic polymorphisms of the human PNPLA3 gene 
are strongly associated with severity of non-alcoholic fatty liver disease in Japanese. PLoS One 2012;7:e38322.

42. Lee SS, Byoun YS, Jeong SH, Woo BH, Jang ES, Kim JW, et al. Role of the PNPLA3 I148M polymorphism in nonalcoholic fatty liver disease and fibrosis in Korea. Dig Dis Sci 2014;59:2967-2974.

43. Park JH, Cho B, Kwon H, Prilutsky D, Yun JM, Choi HC, et al. I148M variant in PNPLA3 reduces central adiposity and metabolic disease risks while increasing nonalcoholic fatty liver disease. Liver Int 2015;35:2537-2546.

44. Anstee QM, Seth D, Day CP. Genetic factors that affect risk of alcoholic and nonalcoholic fatty liver disease. Gastroenterology 2016;150:1728-1744.e7.

45. Buch S, Stickel F, Trépo E, Way M, Herrmann A, Nischalke HD, et al. A genome-wide association study confirms PNPLA3 and identifies TM6SF2 and MBOAT7 as risk loci for alcohol-related cirrhosis. Nat Genet 2015;47:1443-1448.

46. Gao B, Bataller R. Alcoholic liver disease: pathogenesis and new therapeutic targets. Gastroenterology 2011;141:1572-1585.

47. Madrigal-Matute J, Cuervo AM. Regulation of liver metabolism by autophagy. Gastroenterology 2016;150:328-339.

48. Liu X, Green RM. Endoplasmic reticulum stress and liver diseases. Liver Res 2019;3:55-64.

49. Suzuki M, Kon K, Ikejima K, Arai K, Uchiyama A, Aoyama T, et al. The chemical chaperone 4-phenylbutyric acid prevents alcoholinduced liver injury in obese KK-Ay mice. Alcohol Clin Exp Res 2019;43:617-627.

50. Schnabl B, Brenner DA. Interactions between the intestinal microbiome and liver diseases. Gastroenterology 2014;146:1513-1524.

51. Chopyk DM, Grakoui A. Contribution of the intestinal microbiome and gut barrier to hepatic disorders. Gastroenterology 2020;159:849-863.

52. Szabo G. Gut-liver axis in alcoholic liver disease. Gastroenterology 2015;148:30-36.

53. Kitagawa R, Kon K, Uchiyama A, Arai K, Yamashina S, KuwaharaArai $K$, et al. Rifaximin prevents ethanol-induced liver injury in obese KK-Ay mice through modulation of small intestinal microbiota signature. Am J Physiol Gastrointest Liver Physiol 2019;317:G707G715.

54. Chu H, Duan Y, Lang S, Jiang L, Wang Y, Llorente C, et al. The Candida albicans exotoxin candidalysin promotes alcohol-associated liver disease. J Hepatol 2020;72:391-400.
55. Yang AM, Inamine T, Hochrath $\mathrm{K}$, Chen $\mathrm{P}$, Wang L, Llorente $\mathrm{C}$, et al. Intestinal fungi contribute to development of alcoholic liver disease. J Clin Invest 2017;127:2829-2841.

56. Gao B, Radaeva S, Park O. Liver natural killer and natural killer T cells: immunobiology and emerging roles in liver diseases. J Leukoc Biol 2009;86:513-528.

57. Heymann F, Tacke F. Immunology in the liver--from homeostasis to disease. Nat Rev Gastroenterol Hepatol 2016;13:88-110.

58. Tateishi R, Uchino K, Fujiwara N, Takehara T, Okanoue T, Seike M, et al. A nationwide survey on non- $B$, non- $C$ hepatocellular carcinoma in Japan: 2011-2015 update. J Gastroenterol 2019;54:367-376.

59. Tokushige K, Hyogo H, Nakajima T, Ono M, Kawaguchi T, Honda $K$, et al. Hepatocellular carcinoma in Japanese patients with nonalcoholic fatty liver disease and alcoholic liver disease: multicenter survey. J Gastroenterol 2016;51:586-596.

60. Yoshimoto S, Loo TM, Atarashi K, Kanda H, Sato S, Oyadomari S, et al. Obesity-induced gut microbial metabolite promotes liver cancer through senescence secretome. Nature 2013;499:97-101.

61. Yu LX, Schwabe RF. The gut microbiome and liver cancer: mechanisms and clinical translation. Nat Rev Gastroenterol Hepatol 2017;14:527-539.

62. Oze I, Matsuo K, Wakai K, Nagata C, Mizoue T, Tanaka K, et al. Alcohol drinking and esophageal cancer risk: an evaluation based on a systematic review of epidemiologic evidence among the Japanese population. Jpn J Clin Oncol 2011;41:677-692.

63. Yokoyama A, Omori T. Genetic polymorphisms of alcohol and aldehyde dehydrogenases and risk for esophageal and head and neck cancers. Jpn J Clin Oncol 2003;33:111-121.

64. Singh S, Sharma AN, Murad MH, Buttar NS, El-Serag HB, Katzka DA, et al. Central adiposity is associated with increased risk of esophageal inflammation, metaplasia, and adenocarcinoma: a systematic review and meta-analysis. Clin Gastroenterol Hepatol 2013;11:13991412.e7.

65. Go VL, Gukovskaya A, Pandol SJ. Alcohol and pancreatic cancer. Alcohol 2005;35:205-211.

66. Theodoratou E, Timofeeva M, Li X, Meng X, Ioannidis JPA. Nature, nurture, and cancer risks: genetic and nutritional contributions to cancer. Annu Rev Nutr 2017;37:293-320.

67. Marchesini G, Petroni ML, Cortez-Pinto H. Adipose tissue-associated cancer risk: Is it the fat around the liver, or the fat inside the liver? J Hepatol 2019;71:1073-1075. 